\title{
NBIC Collaboration at Multiple Jurisdictional Levels During the Zika Epidemic
}

\author{
Emily larocci*, Collin Schwantes, Anne Folley, Chandra Lesniak, Tiana Garrett-Cherry \\ and Teresa Quitugua
}

Department of Homeland Security, National Biosurveillance Integration Center, Washington, DC, USA

\section{Objective}

An important part of the National Biosurveillance Integration Center's (NBIC) mission is collaboration with federal, state, local, tribal, and territorial governments for the purpose of enhancing early warning, shared situational awareness, and related decision support for infectious disease events. Several such collaborations occurred at multiple jurisdictional levels during the recent Zika epidemic in the Americas and the associated microcephaly and other neurological disorders public health emergency of international concern (PHEIC). The collaborations and their known outcomes from this major infectious disease event are described below, and NBIC stands ready to support similar efforts for future events.

\section{Introduction}

NBIC is charged with enhancing the capability of the Federal Government to enable early warning and shared situational awareness of acute biological events to support better decisions through rapid identification, characterization, localization, and tracking. A key aspect of this mission is the requirement to integrate and collaborate with federal and, state, local, tribal, and territorial (SLTT) government agencies.

NBIC develops and disseminates a variety of products to its stakeholders, including daily reports, ad-hoc reports, analytic collaborations, and leadership briefings upon request. Stakeholders interact with and utilize NBIC's products in different ways, depending on the mission and jurisdiction involved.

Specific collaborations with individual stakeholders are most frequent and evident during major infectious disease events, such as the recent Zika epidemic in the Americas and the associated microcephaly and other neurological disorders PHEIC. Collaborative efforts and known outcomes among varying levels of government are described in detail below in order to highlight NBIC's integration focus and capabilities in this role.

\section{Methods}

NBIC conducted a thorough review of data gathered, reports written, briefings delivered, and both direct and indirect collaborations completed during the Zika epidemic period from late April 2015 March 2017. This review was completed with the intent to document collaborative efforts, feedback, and outcomes with multiple jurisdictions.

\section{Results}

Between April 2015 and March 2017, NBIC worked both directly and indirectly with several of its stakeholders to describe and clarify the Zika event as it unfolded in the Americas. Within the Department of Homeland Security (DHS), NBIC provided briefings for department leadership, including the DHS Secretary and Assistant Secretary of the Office of Health Affairs), and communicated with other DHS components, such as the Federal Emergency Management Agency (FEMA) and the U.S. Coast Guard, to ensure the DHS workforce received effective messaging about Zika infection risks and protections.
For its federal partners, NBIC coordinated and responded to requests for information about Zika across several departments, including the Departments of Defense, State, and Health and Human Services (HHS). In addition, NBIC analysts collected, structured, and provided information about imported and locally-acquired cases described in open source reporting to the HHS Assistant Secretary for Preparedness and Response (ASPR) for inclusion as a layer on the GeoHealth Platform interactive map before Zika reporting was added to the National Notifiable Disease System. Inclusion of this map layer on ASPR's website was NBIC's first public facing collaboration effort. Finally, NBIC coordinated the incorporation of maps and diagrams from ASPR and DOD's Armed Forces Health Surveillance Branch in NBIC products to broaden the distribution of key information.

Outside of the federal government, NBIC received feedback from the Texas Department of State Health Services that a September 2016 NBIC Daily Monitoring List with a map depicting Zika cases near the border of Mexico and Texas, as well as the locations of border crossings, filled a gap in its understanding of the number and distribution of cases in Mexico. State health officials used this information to help target public and clinician outreach activities in Texas. In addition to the regular reports disseminated to the SLTT community, NBIC supported monthly calls for the community to provide infectious disease event updates, including Zika updates, and responded to questions from the community regarding the event and federal government response efforts.

\section{Conclusions}

Major global or regional infectious disease events that have a direct or potential impact on the health of U.S. citizens require substantial collaboration efforts across multiple jurisdictions. These events foster communication and coordination between organizations toward the common goal of serving the American people and keeping them safe and healthy. The Zika epidemic in the Americas and the associated microcephaly/neurological disorders PHEIC is an example of such an event, and NBIC supported its partners and the multiple jurisdictions they serve, as evidenced by the results presented. NBIC continues to expand its network and support capabilities, and is available to serve as integrators for both major and lesser infectious disease events of concern to their stakeholders.

\section{Keywords}

Biosurveillance; National Biosurveillance Integration Center; GeoHealth Platform; Interagency; Zika

\section{*Emily larocci}

E-mail: emily.iarocci@associates.hq.dhs.gov 